\title{
MONITORAMENTO DA ATENUAÇÃO NATURAL DE PLUMA DE CONTAMINAÇÃO PELO MÉTODO DE RADAR DE PENETRAÇÃO NO SOLO (GPR)
}

\author{
César Augusto Moreira e João Carlos Dourado² \\ Recebido em 3 abril, 2006 / Aceito em 5 setembro, 2006 \\ Received on April 3, 2006 / Accepted on September 5, 2006
}

\begin{abstract}
The monitoring of the natural attenuation in contaminated areas has shown an alternative technique of low cost for the remediation of contaminated areas The microorganisms degradation is one of the most important processes in the natural attenuation of contaminants, especially composites of Non-Aqueous Phase Liquid (NAPL). In many cases, the action effective of this process results in generation of organic acid, under high concentrations causes the mineral dissolution presents in the subsurface where if it finds the contamination, with release of ions. The increase on the amount of ions collaborates for the increase of the electric conductivity of the environment. The physical principle of the Ground Penetrating Radar (GPR) is the emission of electromagnetic waves of high frequency. The propagation of the electromagnetic wave depends on the frequency of emitted signal and the electric properties of the subsurface. The increase of electrical conductivity in the subsurface results in the attenuation of the signal and, for consequence, in the reduction of the of penetration depth of electromagnetic wave. This factor allows the monitoring of contaminated areas with natural attenuation from temporal analyses with the GPR. This paper presents a comparative study between profiles of GPR acquired in 1998 and 2003 in an area contaminated for composites of Light Non-Aqueous Phase Liquid (LNAPL), in processes of the natural attenuation. The results indicate an increase of the conductivity in the subsurface, from the accented attenuation it signal GPR observe in the 2003 sections. This increase can be associated to the release of ions for mineral dissolution, for the attack of organic acid resultants of the biodegradation process.
\end{abstract}

Keywords: GPR, monitoring, natural attenuation, biodegradation, hydrocarbons.

RESUMO. 0 monitoramento da atenuação natural em áreas contaminadas tem se mostrado uma técnica alternativa e de baixo custo para a remediação de áreas contaminadas. A degradação por microorganismos é um dos processos mais importantes na atenuação natural de contaminantes, especialmente compostos de fase líquida não aquosa (NAPL). Em muitos casos, a ação efetiva deste processo resulta na geração de ácidos orgânicos, que sob elevadas concentrações ocasionam a dissolução de minerais presentes em subsuperfície onde se encontra a contaminação, com conseqüente liberação de íons. 0 aumento na quantidade de íons colabora para 0 aumento da condutividade elétrica do meio. 0 princípio físico da técnica de Radar de Penetração no Solo (GPR) é a emissão de ondas eletromagnéticas de alta freqüência. A propagação da onda eletromagnética é condicionada à freqüência de sinal emitido e as propriedades elétricas do meio. 0 aumento da condutividade elétrica do meio resulta na atenuação do sinal e, por conseqüência, na diminuição da profundidade de penetração da onda eletromagnética. Este fator permite o monitoramento de áreas contaminadas sob atenuação natural a partir de análises temporais com o GPR. Este trabalho apresenta um estudo comparativo entre perfis de GPR adquiridos em 1998 e 2003 em uma área contaminada por compostos de fase líquida leve não aquosa (LNAPL), sob atenuação natural. Os resultados indicam um aumento da condutividade elétrica do meio, a partir da atenuação acentuada do sinal GPR observada nas seções de 2003. Este aumento pode estar associado à liberação de íons por dissolução de minerais, pelo ataque de ácidos orgânicos resultantes do processo de biodegradação.

Palavras-chave: GPR, monitoramento, atenuação natural, biodegradação, hidrocarbonetos.

\footnotetext{
${ }^{1}$ IGCE, UNESP, Campus de Rio Claro, Av. 24-A, 1515, Bela Vista, Caixa Postal 178, 13506-900 Rio Claro, SP, Brasil - E-mail: cesargeologia@yahoo.com.br ${ }^{2}$ Departamento de Geologia Aplicada, IGCE, UNESP, Campus de Rio Claro, Av. 24-A, 1515, Bela Vista, Caixa Postal 178, 13506-900 Rio Claro, SP, Brasil. Tel.: (19) 3526-2848; Fax: (19) 3534-0327 -E-mail: jdourado@rc.unesp.br
} 


\section{INTRODUÇ̃̃̃O}

A destinação de resíduos industriais é um dos principais problemas ambientais da atualidade, pois muitas vezes não existem opções de reutilização e 0 transporte e disposiçãa em locais adequados demanda elevados investimentos. Este fato tem resultado em ações nocivas, como o descarte indevido direto no solo, com graves conseqüências ao solo atingido e aos recursos hídricos subjacentes.

Dentre as diversas técnicas de remediação de áreas contaminadas, 0 monitoramento da atenuação natural é uma estratégia que pode ser utilizada em determinados casos. Representa um conjunto de processos físicos, químicos e biológicos, que reduzem a massa, toxicidade, mobilidade, volume ou concentração de contaminantes no solo e na água subterrânea sem a intervenção humana. Dentro de condições favoráveis, a atenuação natural pode contribuir significativamente na remediação de contaminações por hidrocarbonetos, podendo ser uma técnica auxiliar e de baixo custo em relação às técnicas convencionais de remediação (EPA, 1998).

Os métodos geofísicos podem auxiliar na detecção e no monitoramento de áreas contaminadas, em conjunto com métodos diretos de investigação, como poços de monitoramento. As técnicas geofísicas de Sondagem Elétrica Vertical (SEV), Radar de Penetração no Solo (GPR) e Eletromagnético Indutivo (EM) tem sido cada vez mais aplicadas para a caracterização e monitoramento de áreas contaminadas por Fase Líquida Não Aquosa (NAPL) (Redman et al., 1994; Benson, 1995; Atekwana et al., 2000).

As vantagens na aplicação do GPR em estudos ambientais residem na possibilidade de execução de perfis contínuos e com alta resolução, facilidade de aquisição de dados, realização de medidas com diversas freqüências e versatilidade do equipamento em campo, possibilitando sua aplicação inclusive am áreas urbanas. Quando comparada com outras técnicas de investigação, este método possui uma baixa relação de custos/benefício aliada à rapidez de execução.

Contudo, a detecção de anomalias depende muito da espessura do solo contaminado, visto que o método apresenta baixa eficiência na detecção de camadas com espessura inferior a 30 a $40 \mathrm{~cm}$ (Redman et al., 1994). Outro fator limitante é a eventual presença de camadas superficiais de alta condutividade, que impedem a penetração dos sinais, resultando em uma menor profundidade de penetração e da resolução do sinal recebido (Benson, 1995).

Este trabalho realiza uma análise comparativa entre seções de GPR obtidos 1998 e 2003, para análise do comportamento de uma pluma de contaminação sob processo de atenuação natural. A influência dos compostos resultantes da degradação da fase contaminante na propagação de ondas eletromagnéticas de alta freqüência também é avaliada.

\section{MATERIAIS E MÉTODOS Localização e histórico da área}

A área está localizada no distrito industrial do município de Araras, estado de São Paulo, Brasil (Fig. 1). 0 local foi ocupado pela Redistil Indústria Química Ltda. entre 1981 e 1985 e pela Sulfabrás S/A entre 1985 e 1992. Dentre os produtos fabricados estão o dieloxalato, acetato de etila, 4-amino-N-2tiozolil-benzeno-sulfonamida, acetopiruvato de metila, 5-metil3-carboxiamidoxazol, e dentre os solventes utilizados durante 0 processo estão 0 1,2 dicloroetano e o tolueno (CETESB, 1997). Os solventes eram reutilizados após passarem por colunas de destilação, e os efluentes gerados eram infiltrados diretamente no solo por meio de um poço de profundidade desconhecida, presente na área. Este procedimento foi utilizado entre 1981 e 1988 (CETESB, 1997).

Em 1988 foi constatada a contaminação do solo e da água subterrânea por 1,2 dicloroetano, benzeno, tolueno, xileno, sódio e cloreto. A partir de 1990 foi dado início ao monitoramento da contaminação pela Companhia de Tecnologia de Saneamento Ambiental (CETESB), por meio de poços de monitoramento, instalados na área industrial e adjacências. Não há dados químicos disponíveis para 0 ano de 2003, visto que as últimas análises dos principais elementos de interesse neste trabalho datam de 1999 (Tab. 1).

0 meio geológico é caracterizado por siltito argiloso, representado no local por solo vermelho escuro argiloso, com matriz de fração silte entre 1 e 3 metros de profundidade e com matriz de fração argila entre 3 e 13 metros de profundidade, com transição gradual entre os níveis. 0 lençol freático raso está situado em profundidades entre 10,7 e 14,1 metros, com variações entre máximas de 7,9 e 14,9 metros. 0 fluxo da água subterrânea nas proximidades dos pontos de infiltração de resíduos é para sudoeste. A condutividade hidráulica varia de $4,4 \times 10^{-7} \mathrm{~cm} / \mathrm{s}$ a $7,0 \times 10^{-7} \mathrm{~cm} / \mathrm{s}$ (CETESB, 1997; Aquino, 2000).

Com 0 fechamento da indústria química em 1992, foi iniciado 0 monitoramento do processo de atenuação natural da pluma contaminante a partir de análises químicas e levantamentos geofísicos, por parte da CETESB.

Em março de 1998 foram realizados levantamentos geofísicos 


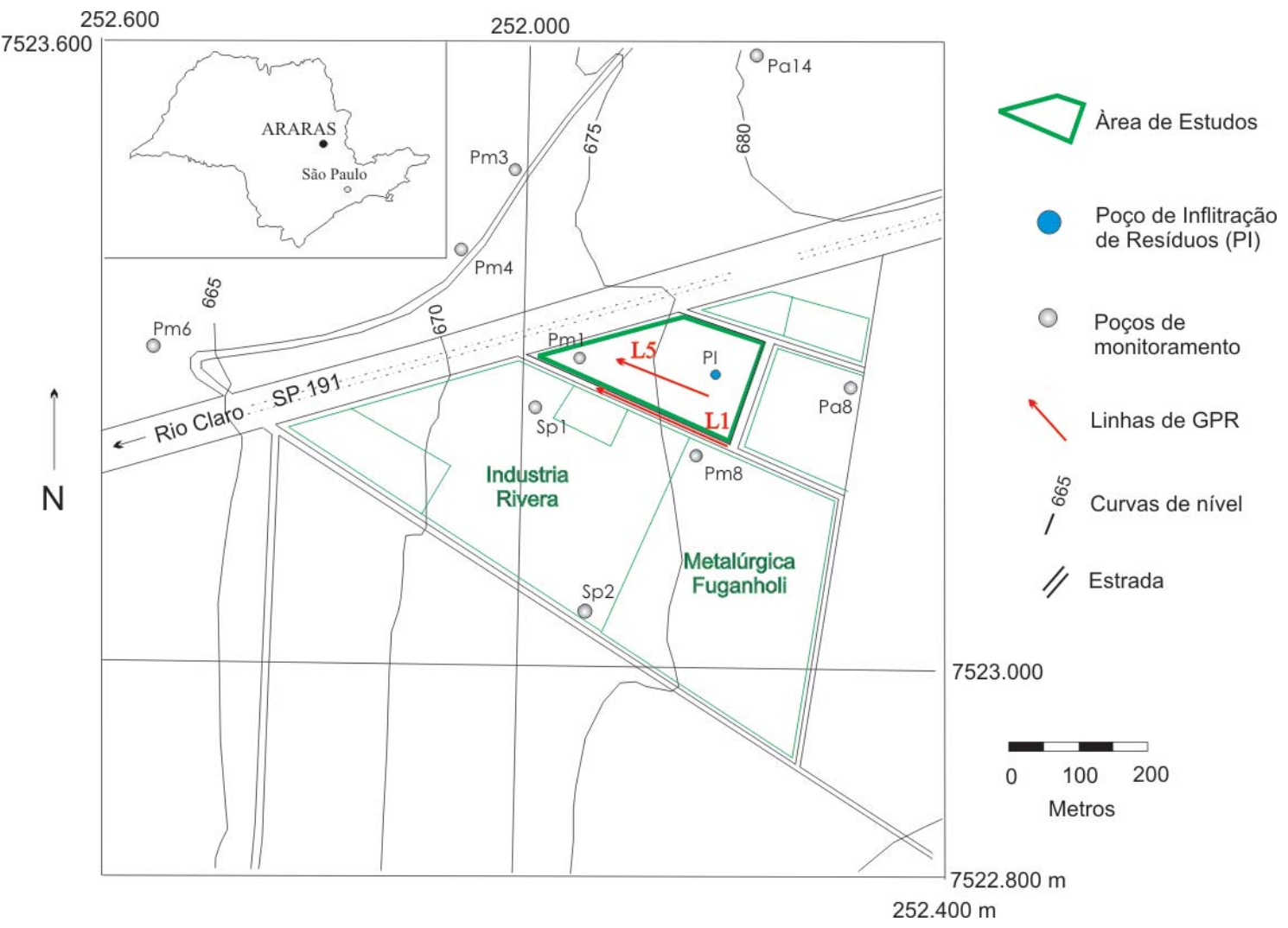

Figura 1 - Mapa de localização da área de estudos, com linhas GPR.

Tabela 1 - Análises químicas do poço de monitoramento da metalúrgica Fuganholi (Pm8).

\begin{tabular}{|l|c|c|c|}
\hline \multicolumn{1}{|c|}{ Contaminante } & $06 / 1990$ & $01 / 1992$ & $11 / 1999$ \\
\hline Benzeno $(\mu \mathrm{g} / \mathrm{L})$ & $29,5 \times 10^{3}$ & $6,0 \times 10^{3}$ & $1,19 \times 10^{4}$ \\
\hline Tolueno $(\mu \mathrm{g} / \mathrm{L})$ & $24,5 \times 10^{3}$ & $7,0 \times 10^{3}$ & $1,39 \times 10^{4}$ \\
\hline 1,2 Dicloroetano $(\mu \mathrm{g} / \mathrm{L})$ & $12 \times 10^{3}$ & 36,0 & 15,9 \\
\hline Sódio $(\mathrm{mg} / \mathrm{L})$ & 200,0 & $2,1 \times 10^{3}$ & - \\
\hline Cloreto $(\mathrm{mg} / \mathrm{L})$ & $2,8 \times 10^{3}$ & $1,8 \times 10^{3}$ & $1,35 \times 10^{3}$ \\
\hline Sulfato $(\mathrm{mg} / \mathrm{L})$ & 32 & 31 & $<10$ \\
\hline
\end{tabular}

por meio de Radar de Penetração no Solo (GPR) (Aquino, 2000). Dentre as conclusões deste trabalho, zonas com ausência de sinal nos perfis de GPR refletem intervalos com elevado grau de contaminação. Estes intervalos apresentam elevada condutividade elétrica e conexão com o poço de injeção de resíduos, definido a partir da aplicação do método Eletromagnético Indutivo na área (Aquino, 2000).

Em março de 2003 foram repetidos os ensaios com GPR nas linhas L1 e L5 de Aquino (2000), segundo os mesmos parâmetros de aquisição e de processamento de dados, com 0 objetivo de análise comparativa, temporal e espacial da contaminação.

\section{Radar de Penetração no Solo}

0 Radar de Penetração no Solo é um método geofísico baseado na propagação de ondas eletromagnéticas de alta freqüência. A partir de uma antena transmissora, são emitidas ondas de energia eletromagnética na faixa de $10 \mathrm{MHz}$ a $1 \mathrm{GHz}$. Quando a onda eletromagnética atinge a interface entre materiais geológicos que apresentam permissividade dielétrica distintas, parte da sua energia refletida em direção à superfície, sendo captada por uma antena receptora (Davis \& Annan, 1989).

A propagação do sinal de radar em subsuperfície depende da 


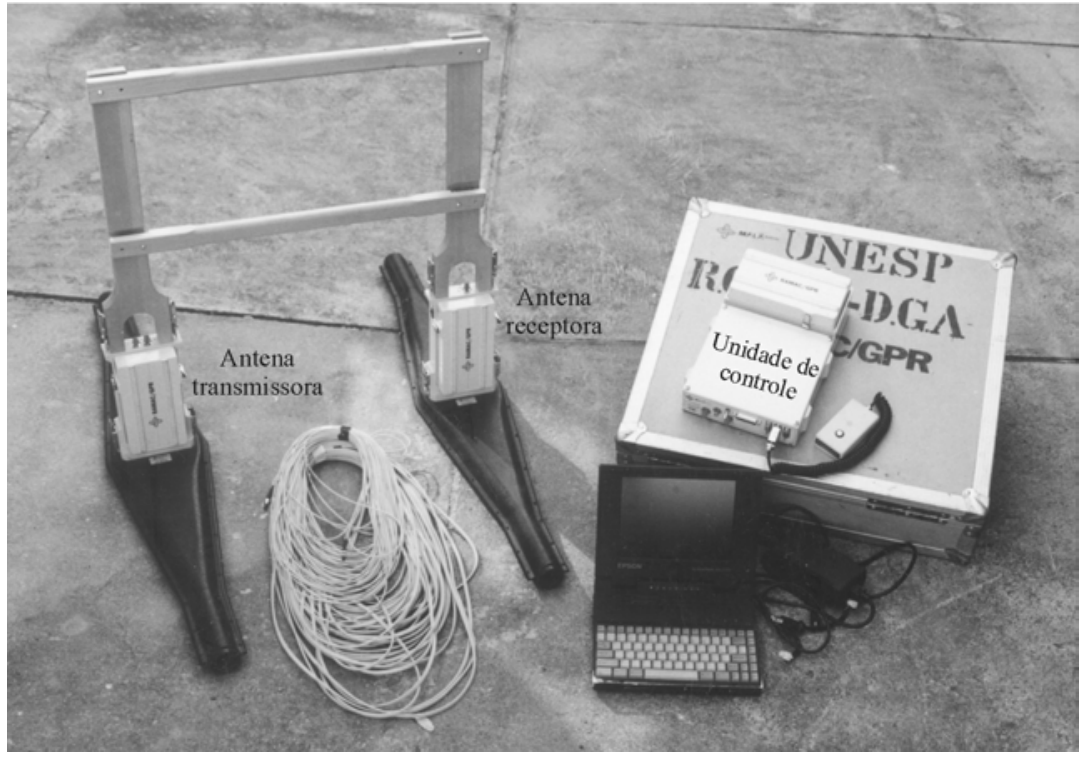

Figura 2 - Equipamento GPR.

freqüência do sinal emitido e das propriedades elétricas do meio. No caso dos materiais geológicos, as propriedades elétricas são controladas principalmente pela mineralogia dos constituintes, presença de argilas, conteúdo de minerais metálicos e pelo conteúdo de água (Beres \& Haeni, 1991).

0 aumento da condutividade elétrica de solos e rochas introduz perdas significativas na propagação do sinal, cujo resultado é a limitada profundidade de penetração.

0 equipamento utilizado foi fabricado pela MÄLA Geoscience (Suécia), com antenas de $100 \mathrm{MHz}$ de freqüência (Fig. 2). As linhas L1 e L5 foram adquiridas em modo passo a passo com intervalos de medida de $100 \mathrm{~cm}$ e $50 \mathrm{~cm}$ respectivamente, com antenas posicionadas de forma transversal à linha de aquisição de dados e espaçadas $60 \mathrm{~cm}$.

Os dados foram processados por meio do programa Gradix, v. 1.08, fabricado pela Interpex Limited (EUA). A conversão tempoprofundidade utilizou 0 valor de velocidade $0,093 \mathrm{~m} / \mathrm{ns}$ para propagação da onda eletromagnético nos materiais geológicos em subsuperfície (Aquino, 2000).

A rotina adotada para o processamento dos dados foi a correção do tempo zero, conversão tempo-profundidade, filtragem DC, ganho AGC e filtragem passa-banda. A aplicação destes parâmetros foi utilizada nos dados de 1998 por Aquino (2000) e pelo caráter comparativo deste trabalho, adotamos os mesmos parâmetros no processamento das seções de 2003.

A filtragem DC é utilizada para remoção de ruídos intrínsecos do equipamento e ruídos de baixa freqüência incorporadas ao traço de aquisição. 0 ganho AGC é um controle de ganho automático utilizado na correção da redução de amplitude do sinal adquirido, devido à atenuação na onda no meio de propagação em subsuperfície. A filtragem passa banda é utilizada com o objetivo de restringir 0 sinal a uma faixa de freqüências próxima ao utilizada pelas antenas de transmissão e recepção, que no presente estudo foi de $100 \mathrm{MHz}$.

\section{Atenuação natural}

0 termo atenuação natural se refere ao conjunto de processos naturais que atuam na remediação de locais contaminados (EPA, 1998). Uma vez determinada a viabilidade do uso desta técnica, deve haver um cauteloso controle e monitoramento do processo.

Um dos fatores mais importantes da atenuação natural é a biodegradação, processo que consiste na mudança da forma dos compostos contaminantes pela ação de microorganismos. Sob condições adequadas, os microorganismos ajudam ou são responsáveis por reações químicas que resultam em compostos que apresentam baixo ou nenhum risco aos seres vivos. Este processo é especificamente importante para a degradação de derivados de petróleo, que podem ser degradados por organismos encontrados em quase todos os ambientes. Vários compostos de fase líquida leve não aquosa (LNAPL), entre eles os BTEX (Benzeno, Tolueno, Etilbenzeno e Xyleno) podem ser biodegradados sob condições ambientais.

Contudo, este processo natural pode ser limitado pela falta de nutrientes, receptores de elétrons ou ambos (Thomas \& Ward, 
1989). Em muitos locais, o processo de bioremediação fica limitado às zonas periféricas da pluma contaminante, pois a presença de contaminante em elevadas concentrações torna 0 ambiente extremamente hostil à proliferação de microorganismos (Wilson \& Jones, 1993).

\section{RESULTADOS}

Tanto as seções adquiridas em 1998 quanto as seções obtidas em 2003 apresentam um intervalo de atenuação do sinal (Figs. $3,4,5$ e 6). Os intervalos de atenuação identificados nas seções estão situados em torno de $2 \mathrm{~m}$ de profundidade, dentro da zona insaturada do perfil de solo. 0 nível situado na profundidade de 4 metros foi adotado como base de comparação para medidas de variações desta porção de sinal atenuado.

A seção L1 de 1998 apresenta dois contatos que limitam 0 intervalo com ausência de sinal, nas posições 100 m e 173 m do início do perfil, totalizando um intervalo de $73 \mathrm{~m}$ (Fig. 3). Os mesmos limites são observados na seção de 2003 (Fig. 4), contudo, deslocados em relação ao perfil anterior. 0 primeiro contato está posicionado a 85 metros do ponto inicial da linha, e 0 segundo contato está posicionado a 185 metros do ponto inicial, totalizando um intervalo de $100 \mathrm{~m}$.

Uma análise comparativa das seções L1 indica um aumento no contraste entre os intervalos de atenuação de 1998 para 2003 (Figs. 3 e 4). A seção de 1998 apresenta estratos horizontais no intervalo atenuado, pouco distinto do meio em redor. A seção de 2003, por sua vez, apresenta forte contraste deste intervalo com o meio em redor, evidenciando aumento na atenuação do sinal.

A seção L5 de 1998 também apresenta um intervalo com atenuação de sinal (Fig. 5), presente desde 0 início da linha até a posição $125 \mathrm{~m}$. Este intervalo se repete na seção L5 de 2003, com o contato posicionado a 135 metros do ponto inicial (Fig. 6).

Também no caso das seções $L 5$, o maior contraste entre 0 intervalo atenuado e o meio em redor ocorre na seção de 2003, em comparação à seção de 1998 (Figs. 5 e 6, respectivamente).

A seção L 5 de 1998 apresenta um intervalo com estratos horizontais dentro da zona de atenuação. É interessante ressaltar que o poço de injeção de resíduos está situado topograficamente acima deste intervalo, com desnível aproximado de $2 \mathrm{~m}$ (Fig. 1).

As hipérboles de difração presentes na seção L1 de 2003 (Fig. 4), entre 85 e 100 metros do ponto de partida, são interferências resultantes de uma nova linha de transmissão de energia instalada no local. 0 deslocamento das camadas da seção a 180 metros do início do perfil nesta mesma seção, é resultado de interferências devido à presença de caçambas metálicas neste ponto.
As interferências responsáveis pelos ruídos nas linhas executadas no ano de 2003 não estavam presentes durante a execução das linhas no ano de 1998.

\section{DISCUSSÃO}

BTEX são hidrocarbonetos aromáticos de baixa solubilidade e elevado coeficiente de partição octanol-água, com concentrações extremamente baixas em água. Contudo, devido à natureza hidrofóbica, estes compostos tendem a se acumular ao redor de partículas de granulometria fina sob a forma de película, com o preenchimento de pequenos poros no intervalo da zona nãosaturada (Newell et al., 1995).

Conforme descrito anteriormente, a propagação da onda eletromagnética em subsuperfície é em grande parte influenciada pela condutividade elétrica do meio. A frente de ondas sofre significativa atenuação ao se propagar em meios com elevada condutividade elétrica, que resulta em reduzidas amplitudes de propagação. Desta forma, o intervalo com ausência de sinais está geralmente associado a um meio que apresenta elevada condutividade elétrica.

Sucessivas análises químicas realizadas em água de poços de monitoramento da área da indústria e adjacências, indicam diminuição na quantidade de contaminantes no meio, inclusive de compostos iônicos (Tab. 1). A explicação para 0 aumento de condutividade elétrica do meio sob este cenário pode estar no processo de degradação dos compostos por ação de bactérias.

Segundo Cerniglia (1984) e Juhasz \& Naidu (2000), as bactérias inicialmente oxidam os hidrocarbonetos aromáticos, cujo produto são cis-dihidrodiols. Este processo envolve a incorporação enzimática de oxigênio atmostérico. A oxidação de cis-dihidrodiols resulta na formação de catechols. Os catechols podem ser oxidados sob a forma orto ou meta (Fig. 7).

0 caminho orto envolve a clivagem da camada entre os átomos de carbono e os dois grupos de hydroxilas, com a produção de cis, cis-ácido muconico. Por outro lado, o caminho meta envolve a clivagem da camada entre os átomos de carbono com os grupos de hidroxilas e os átomos de carbono adjacentes com seu respectivo grupo de hidroxila.

A quebra das cadeias resulta na produção de ácido pirúvico, ácido succinico, ácido fumárico, acido acético e aldeídos, todos utilizados por microorganismos na síntese de constituintes celulares e produção de energia (Wilson \& Jones, 1993). Em conjunto, estas reações produzem dióxido de carbono e água (Fig. 7).

Aparentemente, a degradação de hidrocarbonetos por ação 


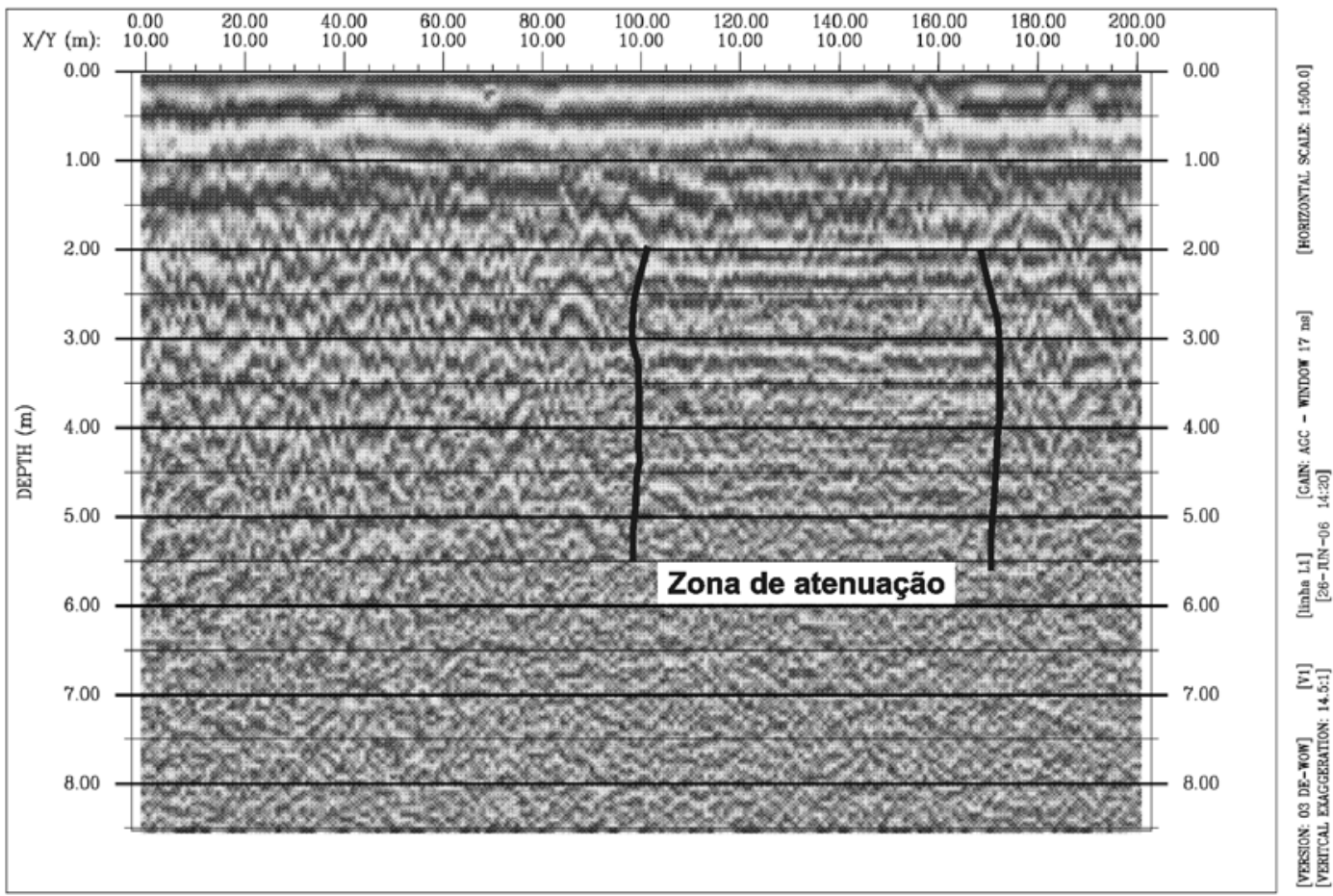

Figura 3 - Linha L1 de 1998.

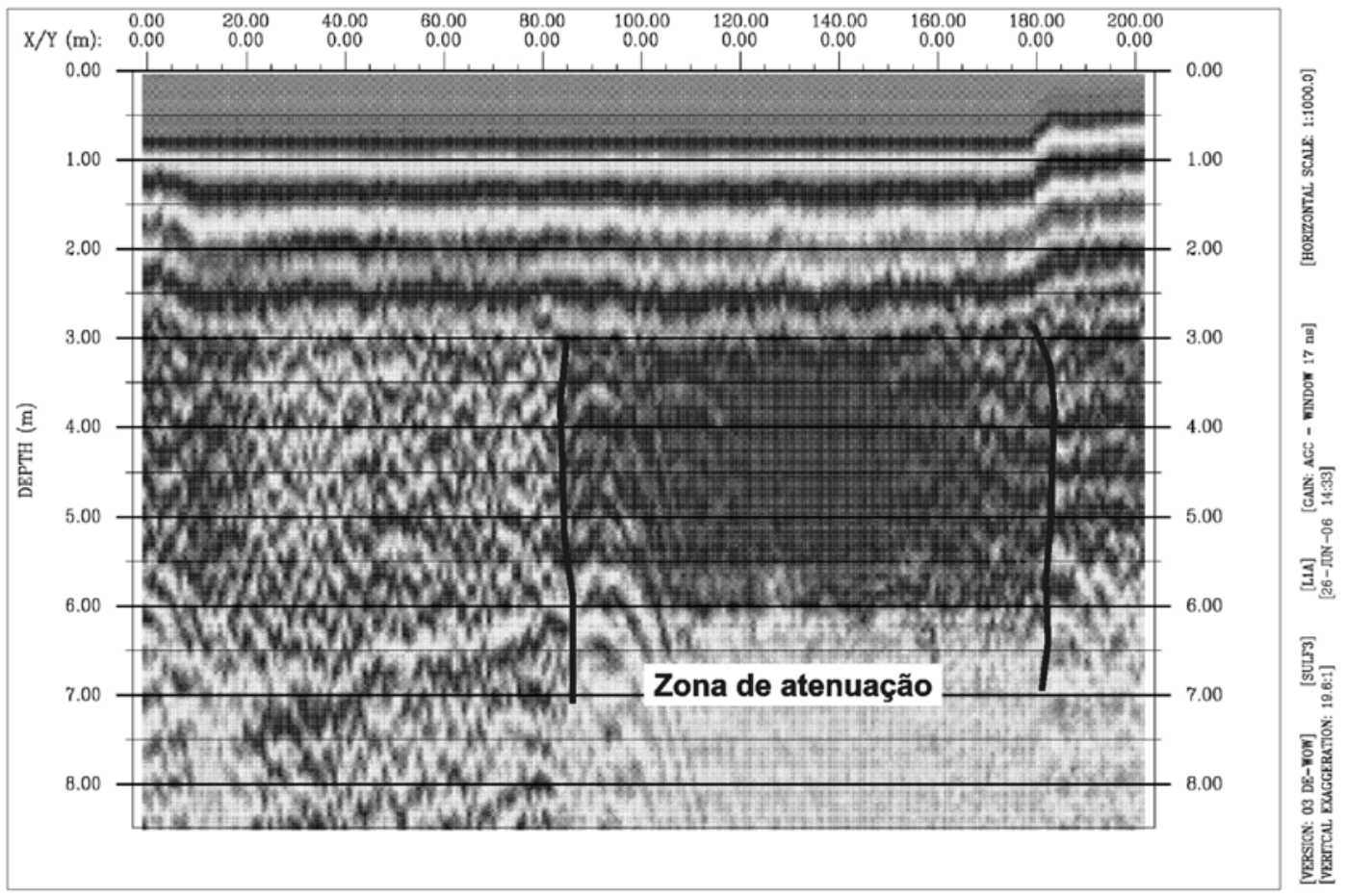

Figura 4 - Linha L1 de 2003. 


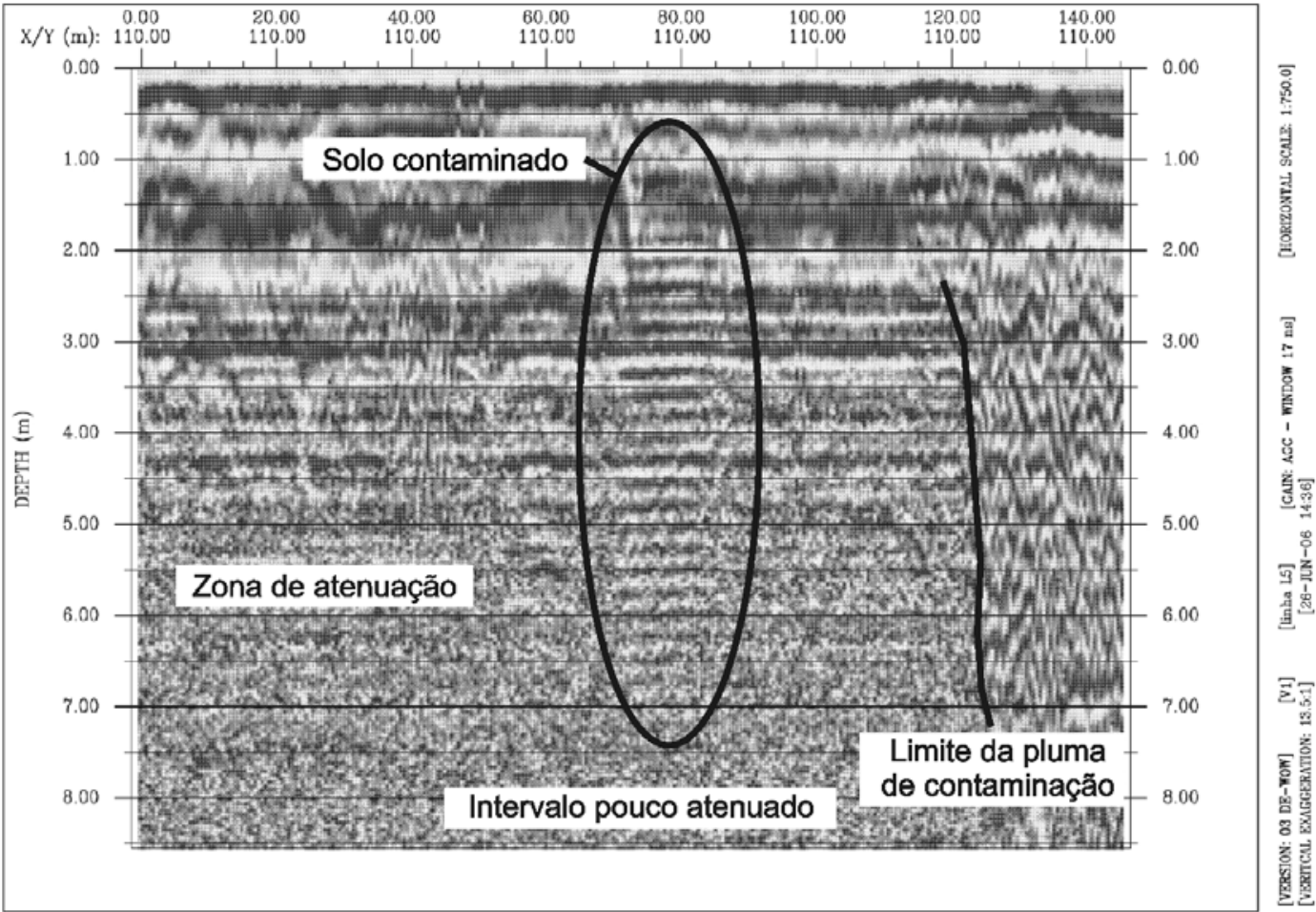

Figura 5 - Linha L5 de 1998

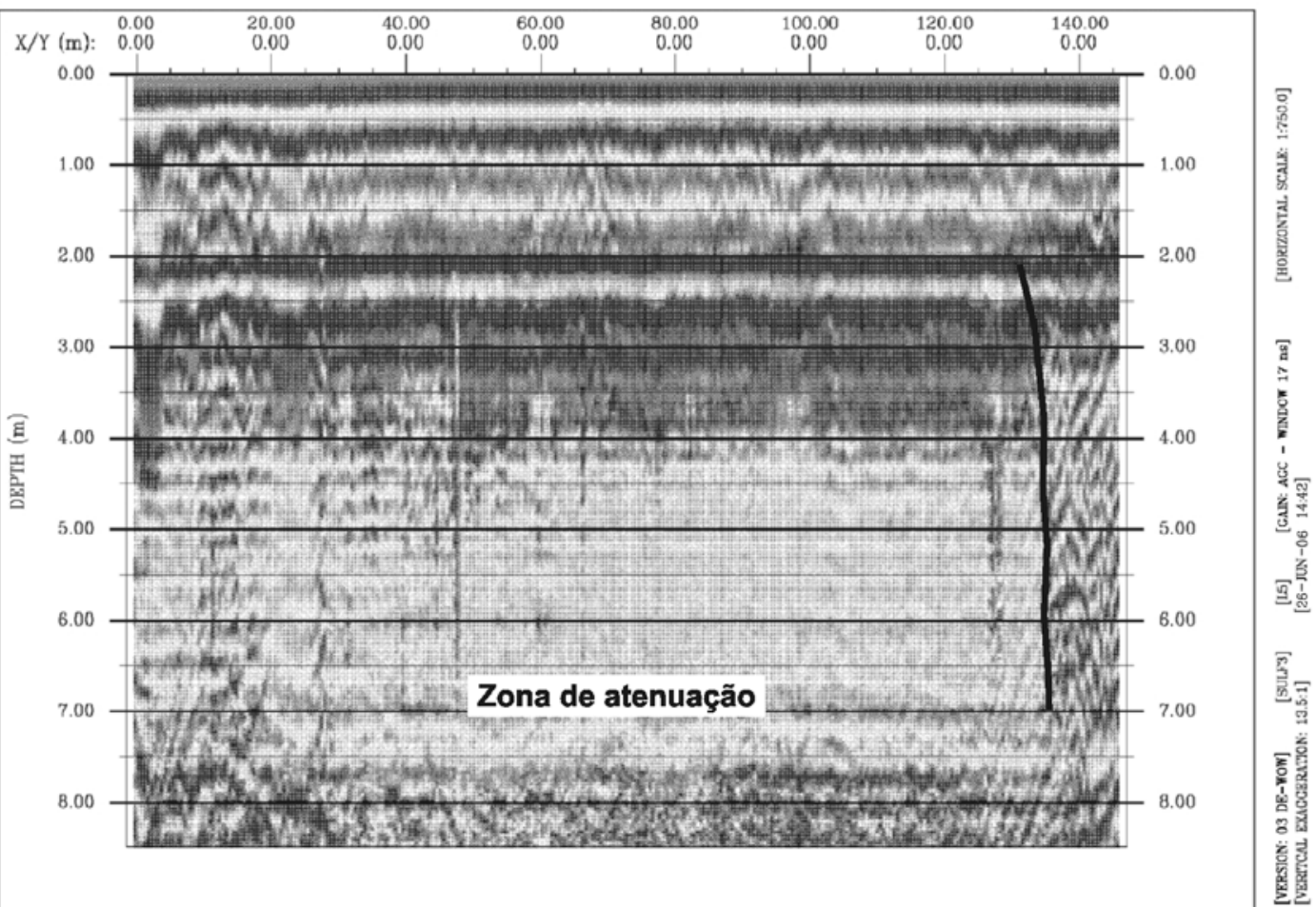

Figura 6 - Linha L5 de 2003 


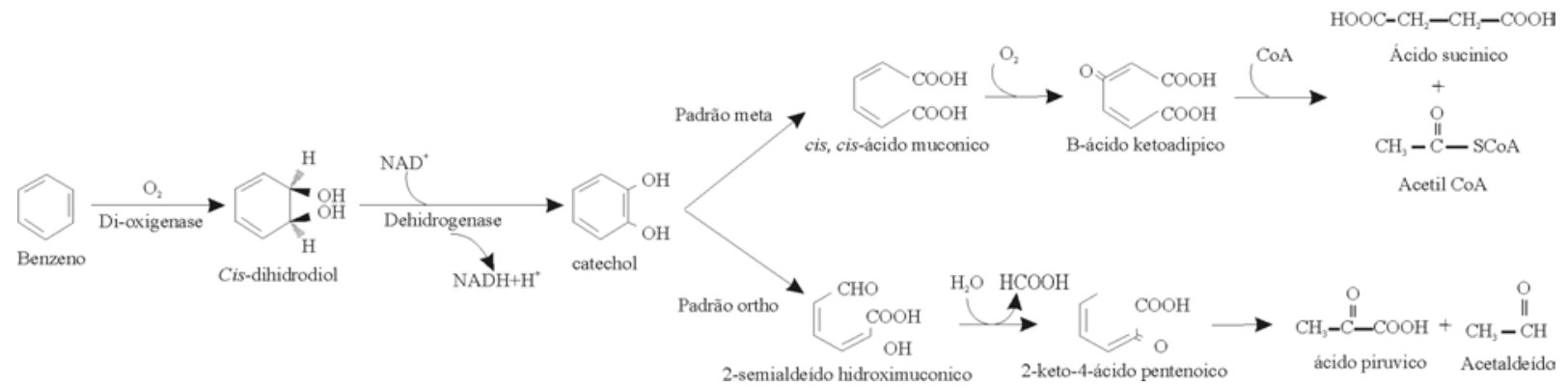

Figura 7 - Metabolismo de microorganismos em cadeias aromáticas segundo o padrão de clivagem orto e meta (adaptado de Juhasz \& Naidu, 2000).

de bactérias na zona insaturada e na zona saturada produzem ácidos carbônico e orgânico, que por sua vez, contribuem para a dissolução mineral dos materiais que constituem 0 aqüífero (McMahon et al., 1995).

A acentuação deste processo resulta na geração de zonas de reação, nas quais a dissolução mineral produz aumento da porosidade, cujos teores podem resultar na supersaturação da fase mineral dissolvida, ou seja, aumento na quantidade de sólidos totais dissolvidos (STD) (Bennett \& Siegel, 1987; Hiebert \& Bennett, 1992).

\section{CONCLUSÕES}

Em ambas as seções foi possível definir intervalos anômalos e de seus limites, caracterizados por elevada condutividade elétrica em relação ao meio em redor. As seções de 2003 apresentam um contraste mais elevado entre intervalo atenuado e meio em redor, em comparação com as seções de 1998.

A comparação entre as seções de GPR indica que a frente de atenuação avançou 12,5 m em média, que resulta em um valor médio de permeabilidade hidráulica de 7,82 × $10^{-6} \mathrm{~cm} / \mathrm{s}$. Este valor está próximo, mas é inferior aos obtidos em ensaios de permeabilidade hidráulica, na ordem de $10^{-7} \mathrm{~cm} / \mathrm{s}$.

Aparentemente não há relação entre o fluxo de água subterrânea, cujo nível varia entre $10 \mathrm{~m}$ e $14 \mathrm{~m}$ de profundidade e a migração de contaminantes na zona insaturada, possivelmente associado à percolação lateral e tendência de fluxo em sentido ao desnível topográfico concomitante à infiltração em profundidade, sentido ao nível freático.

0 fluxo de contaminantes no meio insaturado é bastante complexo, pois ao passo em que a presença de oxigênio intraporos resulta no escape de voláteis, com conseqüente aumento de viscosidade, a degradação de hidrocarbonetos em meio oxidante gera ácidos orgânicos, que em contato com os grãos minerais pode ocasionar mudanças de porosidade e permeabilidade do meio. A compreensão deste fenômeno requer ferramentas de investigação direta, como a instalação de uma rede de poços de monitoramento na área, ensaios de permeabilidade hidráulica em amostras de solo provenientes da zona insaturada e análises químicas para detecção dos produtos de degradação, procedimentos que fogem do escopo deste trabalho.

Os intervalos com ausência de sinal descritos nas seções de GPR são anomalias caracterizadas por elevada condutividade elétrica que resultou na atenuação da onda eletromagnética, aparentemente devido aos produtos e subprodutos da biodegradação de hidrocarbonetos aromáticos provenientes do poço de injeção de resíduos.

A biodegradação contínua dos hidrocarbonetos aromáticos presentes no meio possivelmente resultou no aumento nas concentrações de ácidos orgânicos. A ação de ácidos orgânicos em elevadas concentrações produz dissolução mineral, com aumento da porosidade e elevação nos teores de sólidos totais dissolvidos (STD). É considerável a presença de STD na zona insaturada na área de estudos, visto que o perfil de alteração é caracterizado por siltitos argilosos, ambiente em que o limite entre a zona saturada e insaturada é bastante gradual, acrescido ao fato do mês de março ser chuvoso na região.

A presença de um contraste menor da zona de atenuação da seção L1 de 1998, indica que a condutividade elétrica entre meio atenuado e as adjacências estavam próximas. Em contrapartida, o contraste mais acentuado na seção L1 de 2003 pode indicar que durante o período entre as aquisições em campo houve um aumento considerável na quantidade de STD.

0 intervalo de menor contraste realçado na seção L5 de 1998 é interpretado como uma porção com contaminantes pouco degradados, com características físicas relativamente preservadas, em contraste com o meio em redor, onde o contaminante estaria mais dissolvido, permitindo a degradação por microorganismos, geração de STD e aumento da condutividade elétrica. 
A seção L5 de 2003 não apresenta 0 intervalo realçado na seção L5 de 1998, em contrapartida define um contraste maior entre 0 intervalo de atenuação e o meio em redor, indicativo de ação contínua de processos de degradação de hidrocarbonetos e conseqüente aumento na quantidade de STD durante o período entre 1998 e 2003.

A seção L5 de 2003 em particular foi bastante prejudicada pela presença de ruídos antrópicos instalados posteriormente ao ano de 1998, com geração de hipérboles de difração. Tais ruídos não impediram, contudo, a identificcação de um intervalo atenuado praticamente na mesma posição ao descrito da seção de 1998, com limites comparativamente mais avançados na seção de 2003. Não é possível afirmar a relação direta entre 0 intervalo atenuado como produto da interação da onda eletromagnética com ruídos antrópicos.

0 aumento de condutividade elétrica pode indicar que 0 processo de atenuação natural vigente na área estudada apresenta resultados positivos, com a dispersão dos contaminantes iônicos e degradação dos hidrocarbonetos aromáticos por ação de bactérias com concomitante aumento na quantidade de sólidos totais dissolvidos, que por sua vez reflete diretamente no parâmetro físico condutividade elétrica.

\section{AGRADECIMENTOS}

Este trabalho foi realizado com 0 apoio da Agência Nacional do Petróleo, Gás Natural e Biocombustíveis - ANP e da Financiadora de Estudos e Projetos - FINEP, por meio do Programa de Recursos Humanos para o Setor de Petróleo e Gás - PRH-05ANP/MCT. Os autores agradecem ao técnico Francisco Manuel Garcia Barrera, do Departamento de Geologia Aplicada, UNESP - Rio Claro, pelo auxílio na execução dos trabalhos de campo. Agradecemos também a gentileza do geofísico Wagner França Aquino por ter cedido os dados de GPR referentes ao ano de 1998 e por todo apoio dado à execução deste trabalho.

\section{REFERÊNCIAS}

AQUINO WF. 2000. Métodos Geofísicos Eletromagnéticos aplicados ao diagnóstico da contaminação de Solo e das Águas Subterrâneas em área de Infiltração de Resíduos Industriais. 121 p. (Dissertação Mestrado em Geociências). Instituto de Geociências. Universidade de São Paulo.

ATEKWANA EA, SAUCK WA \& WERKEMA Jr DD. 2000. Investigations of geoelectrical signature at hydrocarbon contaminated site. Journal of Applied Geophysics, 44: 167-180.
BENNETT P \& SIEGEL DI. 1987. Increased solubility of quartz in water due to complexing by organic compounds. Nature, 326: 684-686.

BENSON AK. 1995. Applications of ground penetrating radar in assessing some geological hazards: examples of groundwater contamination. Journal of Applied Geophysics, 33: 177-193.

BERES M \& HAENI FP. 1991. Application of Ground-Penetrating-Radar Methods in Hydrogeologic Studies. Ground Water, 29(3): 375-386.

CERNIGLIA CE. 1984. Microbial Metabolism of Polycyclic Aromatic Hydrocarbons. Advances in Applied Microbiology, 30: 31-71.

CETESB. Companhia de Tecnologia de Saneamento Ambiental. 1997. Relatório do levantamento geofísico para diagnóstico da contaminação da indústria Sulfabrás, São Paulo: CETESB, 91p.

DAVIS JL \& ANNAN AP. 1989. Ground-penetrating radar for highresolution mapping of soil and rock stratigraphy. Geophys. Prospect., 37: $531-551$.

EPA. United States Environmental Protection Agency. 1998. Technical Protocol for Evaluating Natural Attenuation of Chlorinated Solvents in Ground Water. Washington DC, EPA/600/R-98/128. Disponível em: <http://www.epa.gov>. Acesso em: 23 nov. 2003.

HIEBERT FK \& BENNETT PC. 1992. Microbial control of silicate weathering in organic-rich ground water. Science, 258: 278-281.

JUHASZ AL \& NAIDU R. 2000. Bioremediation of high molecular weight polycyclic aromatic hydrocarbons: a review of the microbial degradation of benzo[a]pyrene. International Biodeterioration \& Biodegradation, 45: $57-88$.

MCMAHON PB, VROBLESKY DA, BRADLEY PM, CHAPELLE FH \& GULLETT CD. 1995. Evidence for Enhanced Mineral Dissolution in Organic Acid-Rich Shallow Ground Water. Ground Water, 33(2): 207-216.

NEWELL CJ, ACREE SA, ROSS RR \& HULLING SG. 1995. Light Nonaqueous Phase Liquids. Ground Water Issue, Environmental Protection Agency, Oklahoma, $25 \mathrm{p}$.

REDMAN JD, D'RYCK SM \& ANNAN AP. 1994. Detection of LNAPL pools with GPR: Theoretical modeling and surveys of a controlled spill. In: Proceedings of the Fifth International Conference on Ground Penetration Radar. Ontario, Canada, 3: 1283-1294.

THOMAS JM \& WARD CH. 1989. In situ biorestoration of organic contaminants in the subsurface. Environ. Sci. Technol., 23: 760-766.

WILSON SC \& JONES KC. 1993. Bioremediation of soils contaminated with Polynuclear Aromatic Hydrocarbons (PAHs): a review. Environmental Pollution, 81: 229-249. 


\section{NOTAS SOBRE OS AUTORES}

César Augusto Moreira. Bacharel em Geologia pelo Instituto de Geociências e Ciências Exatas (IGCE) da Universidade Estadual Paulista (UNESP) em 2003. Atualmente é aluno do programa de Pós-Graduação em Geociências e Meio Ambiente do Instituto de Geociências e Ciências Exatas - UNESP - Campus de Rio Claro.

João Carlos Dourado. Bacharel em Geologia pelo Instituto de Geociências da Universidade de São Paulo (USP) em 1977. Doutor em Geociências pela UNESP em 1997. Trabalhou com geofísica aplicada no Instituto de Pesquisas Tecnológicas do Estado de São Paulo de 1977 a 1997. Atualmente é professor assistente doutor do Departamento de Geologia Aplicada do IGCE-UNESP onde atua desde 1997. Trabalha com métodos geofísicos aplicados à Hidrogeologia, Meio Ambiente, Geologia de Engenharia e Geotectônica. 